\title{
Evolution à long terme d'une lande de montagne pâturée par des bovins ou des chevaux. II. Production fourragère
}

\author{
P. Loiseau ${ }^{1}$ et W. Martin-Rosset ${ }^{2}$, \\ avec la collaboration technique de G. Merle ${ }^{1}$ \\ 1/NRA, Station d'Agronomie, F-63039 Clermont-Ferrand; \\ 2 INRA, Station d'Elevage, F-63110 Beaumont, France
}

(reçu le 29-1-1988, accepté le 28-11-1988)

\begin{abstract}
Résumé - On a examiné l'évolution des masses végétales, de la production primaire et des sols sur une lande d'altitude, dans une expérimentation à long terme de remise en exploitation de pâturages montagnards abandonnés. Les chevaux sont plus efficaces que les bovins pour l'élimination des biomasses herbacées ou ligneuses. II en résulte que les fourrages offerts sont plus abondants mais de moindre qualité dans le cas des bovins. La production finale s'établit à 2,5 t MS/ha/an chez les chevaux contre 3,5 chez les bovins. Les matières organiques du sol évoluent au mieux sous les surfaces rasées et piétinées par le cheval. Les chevaux provoquent des transferts et des épuisements locaux en $\mathrm{K}$ alors que les bovins uniformisent la fertilité potassique. L'ensemble des résultats montre, selon les animaux, des évolutions contraires de la valeur botanique et de la productivité. La restitution homogène des fumures réalisée par les bovins est nécessaire au maintien ou à l'accroissement de la productivité; l'élimination des biomasses en excès réalisée par le cheval est suffisante pour l'amélioration botanique. Ceci pose la question de savoir lequel des deux critères doit être privilégié dans une perspective d'entretien pastoral des pâturages de montagne.
\end{abstract}

pâturage de montagne - comportement territorial - comportement alimentaire - cheval de boucherie - Vaccinium myrtillus - Nardus stricta

Summary - Long-term evolution of a hill moor grazed by cattle and horses. II. Forage production. The evolution of the plant biomasses, primary production and soil of a disused hill moor was examined during 12 years after introduction of new management. Horses are more efficient than cattle for the elimination of herbaceous or ligneous masses. As a result, the forage offered is more abundant but of lower quality in the case of cattle. The final production is $2.5 t \mathrm{DM} / \mathrm{ha}$ year by horses versus 3.5 by cattle. The soil organic matter evolves best under the areas closely-grazed and stamped by horses. Horses cause local transfers and exhaustions in $K$, whereas cattle make the K-fertility uniform. According to the animals, the results as a whole, show opposite evolutions of botanical composition and production. The uniform restitution of animal manure due to cattle is necessary for the maintenance or improvement of productivity; the elimination of biomasses in excess due to horses is sufficient for botanical improvement. This raises the question of which of the 2 criteria has to te favored in order to maintain mountain grasslands.

hill moor - territorial behairor - feeding behavior - horse - Vaccinium myrtillus - Nardus stricta

\section{Introduction}

Une expérience de pâturage a été menée pendant 12 ans pour connaître la dynamique des landes montagnards abandonnées lorsqu'elles sont de nouveau soumises à l'action des herbivores domestiques. Le schéma expérimental comprend le pâturage monospécifique par les bovins ou les chevaux (1974-1985), et le pâturage mixte (1979-1985). La conduite en pâturage tournant sur 3 parcelles et sans fertilisation est adaptée à des objectifs d'entretien minimal de la ressource fourragère sans rechercher l'intensification. A ce titre, l'herbivore est le seul outil d'intervention agronomique sur le milieu. Après l'étude de l'évolution botanique, il s'agit ici de décrire et d'expliquer les effets des animaux sur les masses fourragères, notamment sur la production végétale et l'alimentation minérale des peuplements. 


\section{Méthodes}

\section{Protocole expérimental}

Le protocole expérimental est examiné en détail dans un premier article (Loiseau et Martin-Rosset, 1988).

\section{Méthodes}

Dans la suite, il convient de bien distinguer la qualité de l'herbe offerte, base d'un jugement par rapport à l'alimentation du troupeau, de la qualité de l'herbe produite, base d'un jugement de l'effet agronomique du troupeau sur l'alimentation minérale du peuplement.

Herbe offerte. La biomasse épigée offerte au troupeau a été mesurée avant chaque cycle de pâturage sur les parcelles en pâturage monospécifique $1 \mathrm{~B}$ et $1 \mathrm{C}$ en $1978,1979,1980$ et 1982 ainsi que sur les parcelles en pâturage mixte $3 \mathrm{CM}$ et $3 \mathrm{BM}$ en 1980 et 1982. La composition chimique est mesurée par analyse des teneurs en N, P, K, Ca et Mg.

Herbe produite. Elle a été mesurée tous les ans à partir de 1979, en 2 coupes sous cages de mise en défens. Les cages sont installées vers le 20 mai après avoir supprimé les refus. La repousse est mesurée vers le 20 juillet, la cage de nouveau déplacée après nettoyage des refus. La $2^{\theta}$ repousse est mesurée vers le 15 octobre. La première mesure de production a eu lieu à la fin de la première phase expérimentale de pâturage monospécifique sur 6 stations dans chacun des parcs $3 \mathrm{~B}$ et $3 \mathrm{C}$. Les années suivantes, on a ajouté 5 cages dans chacun des parcs $1 \mathrm{~B}$ et $1 \mathrm{C}$ conduits en pâturage monospécifique. Une analyse minérale a aussi été faite sur l'herbe produite.

Etat du sol et enracinement. Une analyse du sol entre 0 et $10 \mathrm{~cm}$ de profondeur a été réalisée en dernière année de pâturage sur les 10 stations en pâturage monospécifique et sur les 12 stations en pâturage mixte. La densité et la biomasse racinaires ont été mesurées par prélèvement à l'emporte-pièce de $0,5 \mid$ dans les tranches de profondeur $0-5,5-10$, $10-15$ et $15-30 \mathrm{~cm}$, et lavage à l'eau sous tamis de $0.5 \mathrm{~mm}$. Les sols ont été analysés pour les teneurs en $\mathrm{C}, \mathrm{N}$ et $\mathrm{P}_{2} \mathrm{O}_{5}$ Dyer, ainsi que pour la capacité d'échange et les teneurs en $\mathrm{K}$, $\mathrm{Ca}$ et $\mathrm{Mg}$ échangeables.

\section{Evolution des biomasses sur pied}

\section{Evolution interannuelle des refus mesurés avant le début d'estive}

La biomasse initiale de $6 \mathrm{t} \mathrm{MS} / \mathrm{ha}$, dont $2 \mathrm{t}$ de bois, décroît au cours du temps pour atteindre un minimum moyen de $1 \mathrm{t}$ en $9^{\mathrm{e}}$ année de pâturage. Par la suite, on note une légère progression avec une moyenne de $2 \mathrm{t}$ de MS/ha en $11^{\mathrm{e}}$ année (Fig. 1a). A moyen terme, les chevaux sont plus efficace que les bovins pour l'élimination des biomasses (Fig. 1a et 1b). Ainsi, en 6 e année, on mesure respectivement 1,8 et 3,3 t MS (parcelles sommitales) et en $11^{\mathrm{e}}$ année 2,1 et 2,8 $\mathrm{t} \mathrm{MS} / \mathrm{ha}$, dont 0,2 et $0,4 \mathrm{t}$ de bois (parcelles de pente). $\mathrm{La}$ variabilité des biomasses en début de saison est toujours plus élevée chez les chevaux.

Après un pâturage mixte, l'effet prépondérant sur l'évolution de la biomasse moyenne est celui

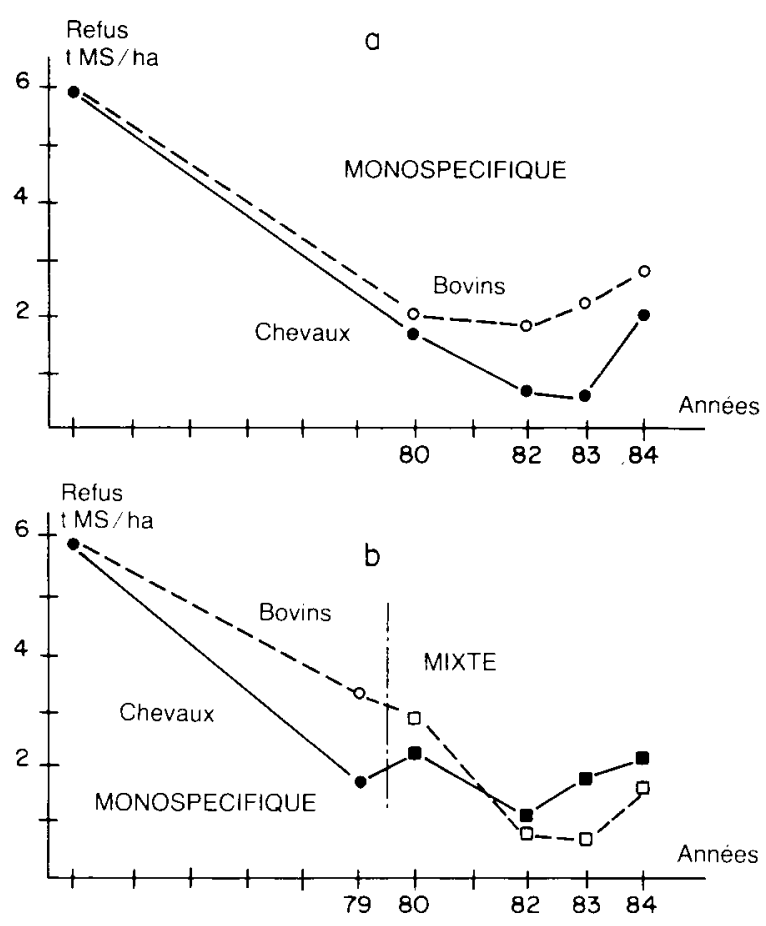

Fig. 1. Evolution des refus présents au début de la saison d'herbe. a. Effet du pâturage monospécifique. b. Introduction de la mixité.

de l'animal nouvellement introduit (Fig. 1b); mais la variabilité des refus dans l'espace reste celle observée précédemment avec le pâturage monospécifique.

\section{Quantités de fourrage offertes aux animaux}

Les quantités de fourrage offertes sont minimum au premier cycle (juin), maximum au $2^{\text {nd }}$ cycle (juillet), et toujours plus importantes sur le pâturage exploité par les bovins. La répartition des masses sur les différentes stations est normale sur les pâturages exploités par les bovins. Sur les pâturages exploités par les chevaux, les stations se répartissent en 2 types distincts : un type "rasé" à $0,5 \mathrm{t} \mathrm{MS} / \mathrm{ha}$ et un type "refusé" à $2,7 \mathrm{t}$ $\mathrm{MS} / \mathrm{ha}$. L'évolution vers les 2 types se réalise en 5 ans à partir d'un état initial des biomasses intermédiaires de 1,5 t MS/ha (Fig. 2a). Sur les stations rasées, la biomasse diminue au cours de la saison; sur les stations refusées, la biomasse se stabilise vers $3 \mathrm{t} \mathrm{MS} / \mathrm{ha}$ à partir du mois de juillet (Fig. 2b).

\section{Qualité des fourrages offerts}

La teneur en azote du fourrage offert décroît de 0,4 points au cours de la saison quel que soit le troupeau pâturant, mais la concentration en azote reste toujours plus élevée chez les chevaux (Fig. 3). Les teneurs élevées sur les pâtu- 

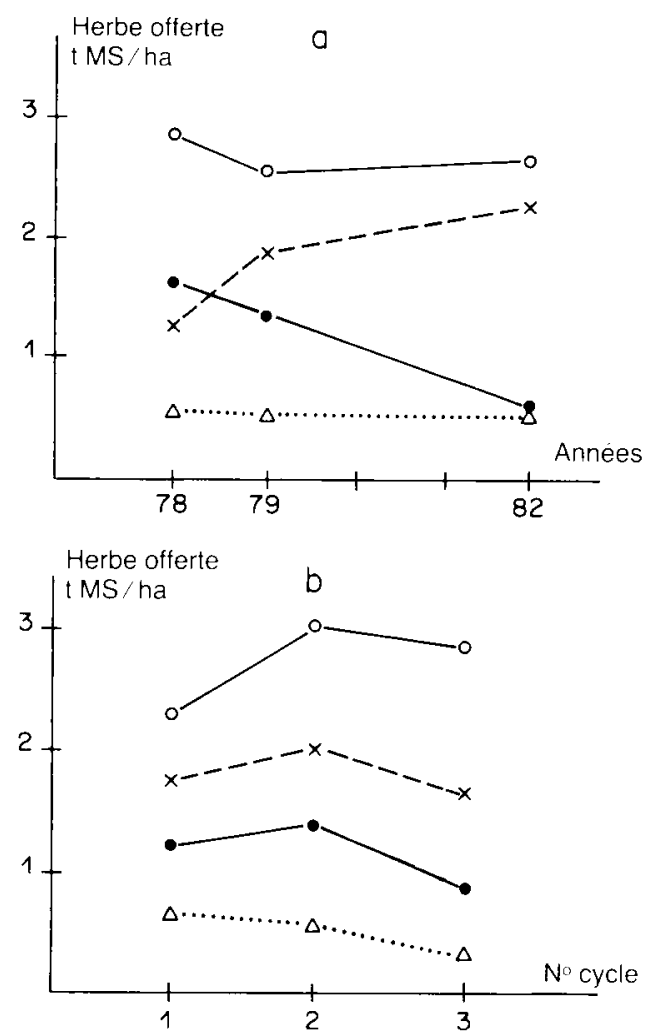

Fig. 2. Variation des quantités de fourrage offertes aux chevaux selon les stations. a. Evolution interannuelle. b. Evolution intraannuelle. $\mathrm{O}-\mathrm{O}$ : stations refusées $\left(n^{\circ} \mathrm{s} 8,9,10\right)$; $\Delta--\Delta$ : stations rasées (nos $1,5,6$, ); $X-X$ : station intermédiaire (nos 7 ), $\bullet . . \bullet$ : stations intermédiaires (nos $2,3,4$ ).

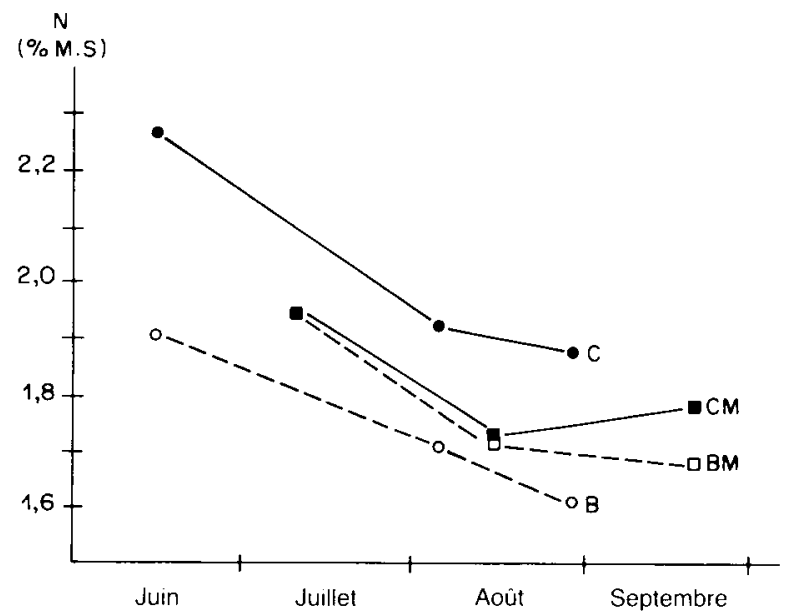

Fig. 3. Effet du pâturage monospécifique ou mixte sur la qualité azotée du fourrage offert (moyenne de 2 années: 1980 et 1982). C : chevaux; B : bovins; CM : chevaux, puis mixte; $\mathrm{BM}$ : bovins, puis mixte.

rages exploités par les chevaux sont principalement attribuables aux stations rasées. Chez les bovins, les teneurs faibles sont liées à des biomasses élevées et à une maîtrise insuffisante de l'épiaison des graminées.

Les teneurs dans les autres éléments restent peu affectées par la saison ou le type d'animal, à l'exception du potassium dont la teneur est extrêmement faible et en diminution pendant l'année de pâturage.

Sur les 3 premières années de mixité, la qualité des fourrages offerts devient intermédiaire entre celle des 2 pâturages monospécifiques. Après un pâturage bovin, l'introduction du cheval améliore la teneur moyenne de 0,1 point en moyenne (Fig. 3).

\section{Evolution de la production primaire en 12 ans}

\section{Pâturages monospécifiques}

Au cours des 10e (1983) et $11^{\mathrm{e}}$ (1984) années de pâturage, les productions moyennes sont significativement plus élevées de $1 \mathrm{t}$ de MS/ha chez les bovins que chez les chevaux (Tableau I). La production des stations témoins non pâturées reste intermédiaire avec 3,2 t MS/ha.

La variabilité entre stations double au cours des années pour les deux troupeaux. Chez le cheval, l'accroissement de la variabilité correspond à l'appauvrissement de certaines stations dont la production diminue de moitié en 5 ans par rapport à la production moyenne initiale (Fig. 4c). Chez les bovins, l'accroissement de la production et de la variabilité résulte d'une amélioration de $50 \%$ de la production sur une seule station (Fig. 4b).

\section{Pâturages mixtes}

L'introduction des bovins sur le pâturage exploité d'abord par les chevaux favorise temporairement la production en $2^{e}$ année de mixité, mais au bout de 5 années, la production diminue fortement par rapport à celle du pâturage bovin monospécifique. De même, l'introduction de chevaux sur le pâturage exploité par les bovins entraîne une diminution constante de la production qui perd le quart de sa valeur en 5 ans. A la fin de l'expérimentation, la production des parcelles où ont pâturé des chevaux est de 73 à $77 \%$ de celle du pâturage bovin, aussi bien en pâturage monospécifique que mixte, et quel que soit le précédent (Fig. 4a).

La variabilité entre stations induite initialement par le cheval se poursuit pendant la période de mixité (Fig. 4e) : la diminution de production affecte aussi bien les stations initialement les plus pauvres ou les plus riches. L'introduction du cheval après pâturage monospécifique par les bovins crée un début d'hétérogénéisation (Fig. 4d). L'effet du cheval reste donc de tous les points de vue prédominant sur l'effet des bovins. 
Tableau I. Production en matière sèche des pâturages selon l'herbivore et les années de pâturage ( $\mathrm{kg} / \mathrm{ha} / \mathrm{an})$.

\begin{tabular}{lllllll}
\hline & 1980 & 1981 & 1982 & 1983 & 1984 & Moy. \\
\hline Chevaux (C) & $3554 \mathrm{a}$ & $2557 \mathrm{a}$ & $3265 \mathrm{a}$ & $2661 \mathrm{a}$ & $2522 \mathrm{a}$ & $2912 \mathrm{a}$ \\
Bovins (B) & $3525 \mathrm{a}$ & $2920 \mathrm{a}$ & $3718 \mathrm{a}$ & $3678 \mathrm{~b}$ & $3476 \mathrm{~b}$ & $3464 \mathrm{~b}$ \\
Mixte (CM) & $3456 \mathrm{a}$ & $3219 \mathrm{~b}$ & $3387 \mathrm{a}$ & $3607 \mathrm{ab}$ & $2695 \mathrm{a}$ & $3279 \mathrm{bc}$ \\
Mixte (BM) & $3506 \mathrm{a}$ & $2811 \mathrm{a}$ & $3251 \mathrm{a}$ & $3249 \mathrm{ab}$ & $3579 \mathrm{a}$ & $3079 \mathrm{ac}$ \\
Moyenne & $3510 \mathrm{z}$ & $2877 \mathrm{x}$ & $3405 \mathrm{z}$ & $3299 \mathrm{z}$ & $2818 \mathrm{x}$ & 3183 \\
\hline
\end{tabular}

Les productions affectées d'une même lettre $(a, b, c)$ ne sont pas significativement différentes au niveau $1 \%$.
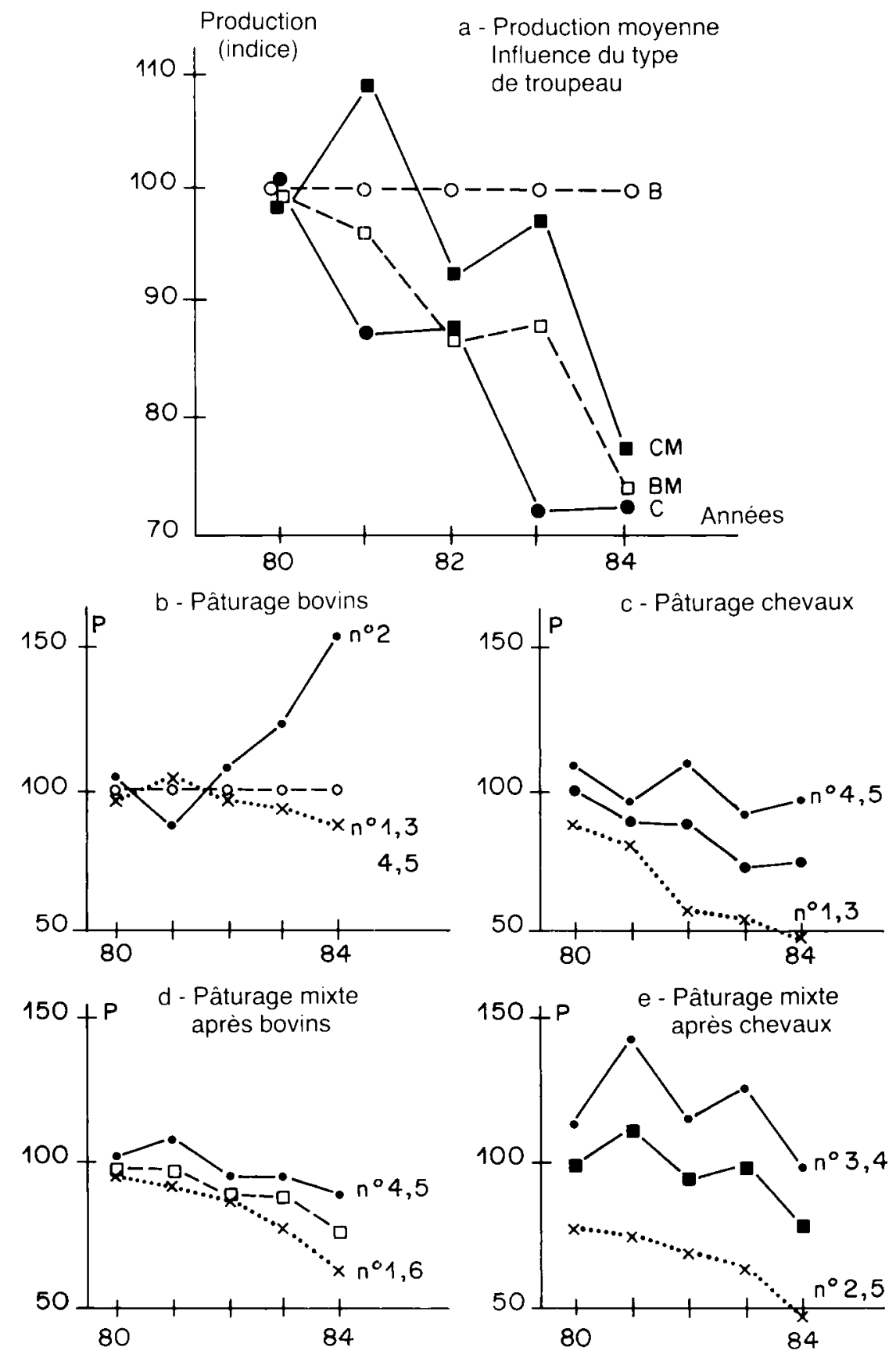

Fig. 4. Evolution de la production végétale. La production du pâturage bovin est à l'indice 100 pour chaque année. Pour les valeurs absolues, se reporter au Tableau l. a. Production moyenne; influence du troupeau. b, c, d, e. Production par type de station. b. bovins (moyenne de 5 stations); c. chevaux (moyenne de 5 stations); $d$. chevaux, puis mixte (moyenne de 6 stations); e. Bovins, puis mixte (moyenne de 6 stations). Les chiffres renvoient à la moyenne de stations particulières dont les numéros sont indiqués. 


\section{Alimentation minérale des pâturages}

Les teneurs en minéraux de l'herbe produite récoltée en cages de mise en défens (Tableau II) montrent par rapport aux normes d'interprétation de De Montard (1987) une nutrition insuffisante en phosphore et, surtout, en potassium. Des spécificités apparaissent en fonction de l'herbivore. Chez les chevaux, la production est corrélée positivement avec la teneur en potassium qui est fortement variable ( $\mathrm{CV}$ de $27 \%$ ) et négativement avec la teneur en magnésium (Tableau III). Chez les bovins, les teneurs en potassium varient moins (CV de 19\%), et la production est liée positivement aux teneurs en calcium. On peut donc considérer que les bovins effacent les hétérogénéités de disponibilité en potassium en répartissant cet élément de façon homogène sur leur territoire, et laissent s'exprimer un critère de fertilité ancien ou acquis, lié au calcium. Quant aux chevaux, ils ne font qu'exacerber la variabilité de la fertilité potassique par leur propre comportement.

A partir de la $9^{e}$ année de pâturage, les quantités d'éléments minéraux mobilisés dans la croissance de l'herbe sont très comparables entre l'ensemble du pâturage bovin et les surfaces refusées par les chevaux (Tableau II). En revanche, les surfaces très pâturées par les chevaux mobilisent des quantités d'éléments largement inférieures (Archer, 1973). Elles se distinguent aussi par une forte diminution du rapport $\mathrm{K} / \mathrm{N}$ et une compensation du déficit en bases par l'utilisation accrue du magnésium. Le problème reste de savoir si la faible production des surfaces très pâturées par les chevaux résulte d'un effet direct de l'animal sur la croissance végétale, ou d'un effet indirect sur la croissance par le biais de l'alimentation minérale des végétaux.

\section{Discussion : évolution de la production et de la valeur pastorale}

L'interprétation des résultats moyen d'évolution végétale sur les parcelles, aussi bien en composition botanique (Loiseau et Martin-Rosset 1988) qu'en production fourragère ramène à considérer le fonctionnement écologique du système solpeuplement-troupeau à l'intérieur de la parcelle. L'évolution moyenne sous l'influence du troupeau résulte de comportements animaux spécifiques qui peuvent être répartis de façon homogène ou,

Tableau II. Mobilisation des éléments minéraux dans la production de matière sèche $(\mathrm{kg} / \mathrm{ha} / \mathrm{an})$ et nutrition des peupiements.

$\begin{array}{lllllllll}N & K & P & C a & M g & K / N & P / N & C a / N & \begin{array}{l}M g / K \\ (k g)\end{array} \\ (k g) & (k g) & (k g) & (k g) & (\%) & (\%) & (\%) & (\%)\end{array}$

\begin{tabular}{|c|c|c|c|c|c|c|c|c|c|}
\hline \multicolumn{10}{|l|}{ Chevaux } \\
\hline Zones paturées & 49,5 & 17,2 & 3,2 & 7,6 & 4,6 & 35 & 6 & 15 & 27 \\
\hline Zones refusées & 71,4 & 38,0 & 5,1 & 14,7 & 6,4 & 53 & 7 & 21 & 23 \\
\hline Bovins & 74,8 & 38,7 & 5,6 & 14,7 & 6,2 & 52 & 7 & 20 & 16 \\
\hline
\end{tabular}

Moyenne des années 9 à 11.

Tableau III. Signe des corrélations entre teneurs minérales du fourrage produit et production.

\begin{tabular}{|c|c|c|c|c|c|c|c|c|c|c|}
\hline & \multicolumn{5}{|c|}{ Bovins } & \multicolumn{5}{|c|}{ Chevaux } \\
\hline & $\% N$ & $\% P$ & $\% K$ & $\% \mathrm{Ca}$ & $\% M g$ & $\% N$ & $\% P$ & $\% K$ & $\% \mathrm{Ca}$ & $\% M g$ \\
\hline \multicolumn{11}{|l|}{$\% N$} \\
\hline \multicolumn{11}{|l|}{$\% P$} \\
\hline$\% \mathrm{~K}$ & & + & & & & & + & & & \\
\hline \multicolumn{11}{|l|}{$\% \mathrm{Ca}$} \\
\hline$\% \mathrm{Mg}$ & & & & + & & & + & & & \\
\hline Prod. & & & & + & & & & + & & - \\
\hline
\end{tabular}

+ : Corrélations significatives à un niveau de $5 \%$ 
au contraire, dissociés selon les stations à l'intérieur de la parcelle. On distingue d'une part l'action physique liée au prélèvement du fourrage (effets de coupe et de piétinement), d'autre part le transfert et le recyclage des éléments restitués (effet fumure).

Pour les bovins, on peut considérer que le troupeau assure une sélection alimentaire et une répartition des excrétats homogènes. Pour les chevaux, la restitution sans coupe est opposée à la coupe sans restitution; ce comportement dual et sans appel est rapporté dans la littérature, même dans le cas de végétations initialement homogènes. Nous constatons comme Edward et Hollis (1982) la permanence presque parfaite dans le temps de ce comportement sur les mêmes surfaces.

\section{Production et fertilité du sol}

II est déjà connu que, pour des végétations dégradées du domaine montagnard, une augmentation du chargement en pâturage tournant provoque une augmentation de la production grâce au recyclage accéléré des éléments minéraux à travers le tube digestif des herbivores (INRA, 1979). Ce résultat obtenu avec les ovins sur nardaie est confirmé ici avec les bovins sur lande. En accord avec Archer (1977), nous constatons que le comportement des bovins efface les hétérogénéités de disponibilité en potassium en répartissant cet élément de façon homogène sur leur territoire. L'augmentation de fertilité se traduit surtout par une meilleure disponibilité en bases (Fig. $5 b$ ) et une diminution du C/N (Fig. 5a). A plus forte raison, la production est aug-
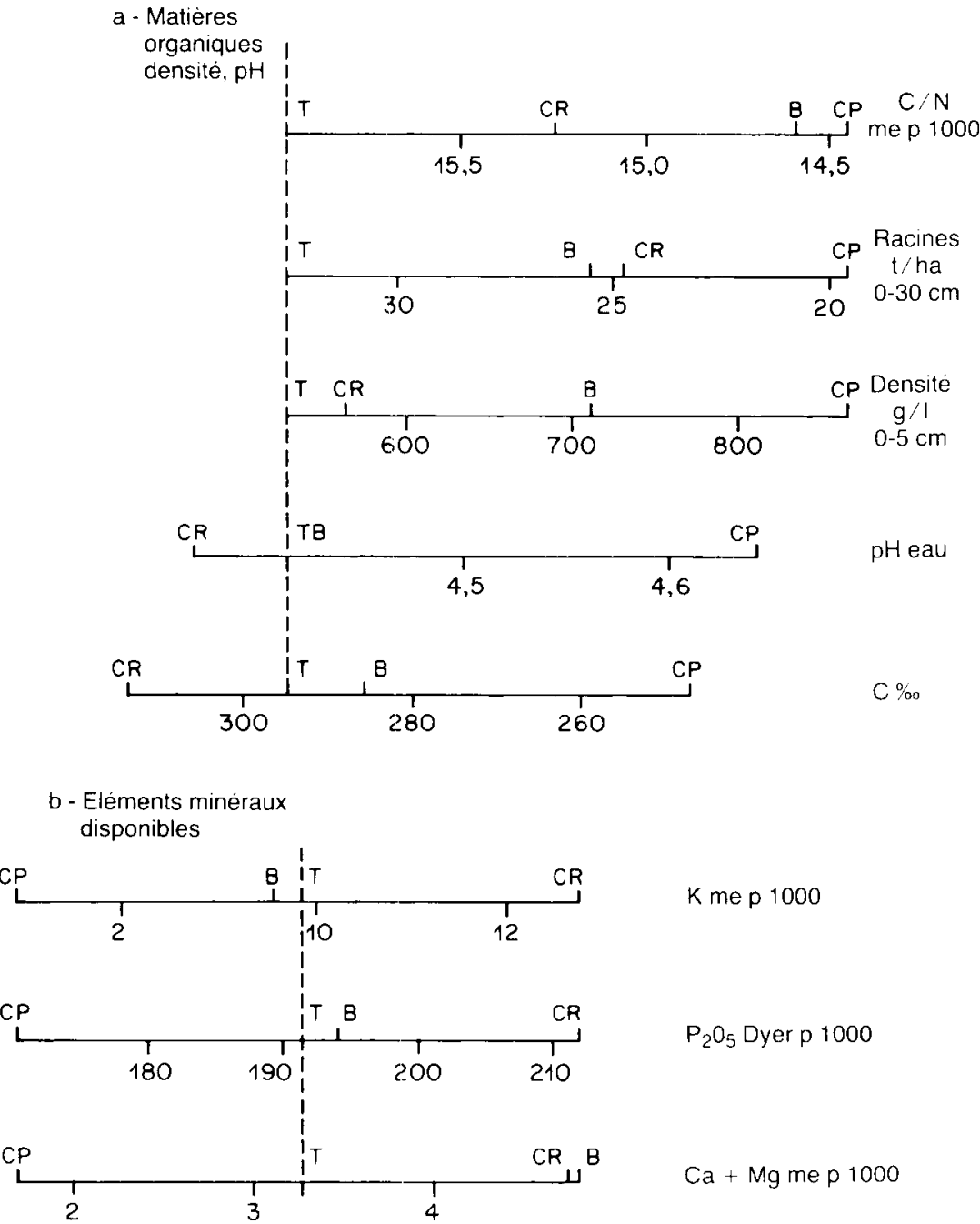

Fig. 5. Evolution du sol. Etat en $12^{e}$ année (analyses sur $0-5 \mathrm{~cm}$, sauf indications). Les échelles sont choisies pour donner le même poids à chaque descripteur. $T$ : témoin de l'état initial; $B$ : bovins; $C R$ : chevaux, faciès refusé et fertilisé; $C P$ : chevaux, faciés pâturé sans restitution. 
mentée dans les pâturages de chevaux sur les aires qui bénéficient d'un transfert positif de fumure.

Inversement, les surfaces rasées, piétinées et non fertilisées par les chevaux subissent une forte chute de production. Le pâturage ras peut en effet entraîner une chute de la productivité par différentes actions liées à ce comportement : mauvais enracinement dû aux coupes fréquentes et au piétinement (Fig. 5a), réduction de la photosynthèse par celle de l'indice foliaire, épuisement chimique par transfert de fumure vers les zones refusées (Fig. 5b). Les résultats montrent que ces 3 facteurs sont en cause. La masse photosynthétisante reste inférieure à $1 \mathrm{t}$ MS pendant toute la saison de croissance (Fig. 2b); la masse racinaire est diminuée (Fig. $5 \mathrm{a}$ ); la dégradation de la nutrition potassique, constatée par l'analyse des matières sèches produites (Tableau II), est confirmée par l'analyse des sols (Fig. 5b).

Cependant, les excès de coupe et de piétinement sur les surfaces rasées par le cheval n'ont pas que des aspects négatifs. Dans des milieux initialement engorgés par des excès de matières organiques, les sols prélevés sous les surfaces rasées présentent une évolution très favorable du point de vue de l'état de ces matières organiques (Fig. 5a) : diminution des teneurs en carbone de 30 à $25 \%$, augmentation de la densité de 550 à $850 \mathrm{~g} / \mathrm{l}$, diminution du $\mathrm{C} / \mathrm{N}$ de 16 à 14,5. En effet, les apports organiques sont limités (diminution de la production, consommation de l'intégralité de la biomasse épigée et absence de déjections). D'autre part, l'augmentation de la densité du sol, qui pourrait être néfaste dans d'autres milieux (Rogalsky 1978), améliore les conditions physiques nécessaires à l'évolution des matières organiques libres. Le tout converge vers un désengorgement de la chaîne catabolique des matières organiques dans l'écosystème : les sols sous les surfaces rasées seraient donc améliorés du point de vue de leur potentiel de minéralisation de l'azote mais incapables d'exprimer ce potentiel par la limitation de l'indice foliaire et de la fertilité potassique.

\section{Evolution simultanée de la composition bota- nique et de la production}

Chez les bovins (Fig. 6a), les modalités de prélèvement du fourrage ne sont pas défavorables pour la production mais n'entraînent pas d'amélioration botanique en raison de la sélection alimentaire des meilleures espèces fourragères (Carson et Wood-Gush, 1983). Une évolution se produit à valeur pastorale constante vers une pelouse médiocre mais relativement productive à Nardus stricta.
Chez les chevaux (Fig. 6b), le piétinement et la coupe répétée améliorent fortement l'état des matières organiques et la composition botanique au profit de Festuca rubra et Agrostis tenuis, mais diminuent aussi beaucoup la production primaire. A elles seules, les déjections ne provoquent guère d'amélioration botanique bien qu'elles augmentent beaucoup la production et la fertilité chimique du sol.

II en résulte que, sur la lande, la restitution des fumures est une condition nécessaire au maintien ou à l'accroissement de la production, et que la coupe intégrale est une condition suffisante pour l'amélioration botanique. Les lois d'action des différents effets animaux sont légèrement différentes dans le cas des pelouses oligotrophes (Loiseau et Martin-Rosset, 1985).

\section{Cas du pâturage mixte}

Lorsqu'on associe les bovins aux chevaux, les différents effets animaux se manifestent toujours dans le cadre de la dualité territoriale imposée par le cheval. Conformément à Archer (1980), on observe une consommation par les bovins des refus laissés par les chevaux sur leurs latrines. L'indice de valeur pastorale s'y améliore, vérifiant en cela le principe de Carson et Wood-Gush (1983) selon lesquels une complémentarité des comportements alimentaires améliore la composition botanique. La VP s'accroît de 17 à 28 (Fig. $6 d)$, sans toutefois atteindre la valeur de 40 obtenue sur les aires de pâturage des chevaux (Fig. $6 b)$.

En revanche, l'introduction du bovin n'améliore pas les surfaces rasées par les chevaux (Fig. 6c) : la mixité ne permet pas spécialement, comme le pense Arnold (1980), une meilleure répartition des déjections. Conformément aux observations de Edward et Hollis (1982), les ruminants sont confinés sur les latrines des chevaux et y restituent la plupart de leurs propres déjections. Sauf exception (proximité du point d'eau), il n'y a donc pas de modifications importantes dans la localisation des fumures animales. Seule la suppression des chevaux et leur remplacement par des bovins pendant au moins un an (Archer, 1980) pourrait contribuer à homogénéiser et améliorer la production des pâturages à chevaux (sans améliorer au-delà la composition botanique).

En conclusion, l'intérêt de l'association des bovins sur un pâturage à chevaux est d'abord dans la meilleure valorisation des fourrages disponibles, et secondairement dans l'amélioration botanique des latrines. Dans l'autre sens, l'introduction du cheval sur un pâturage à bovins corrige les effets néfastes du pâturage sélectif des 

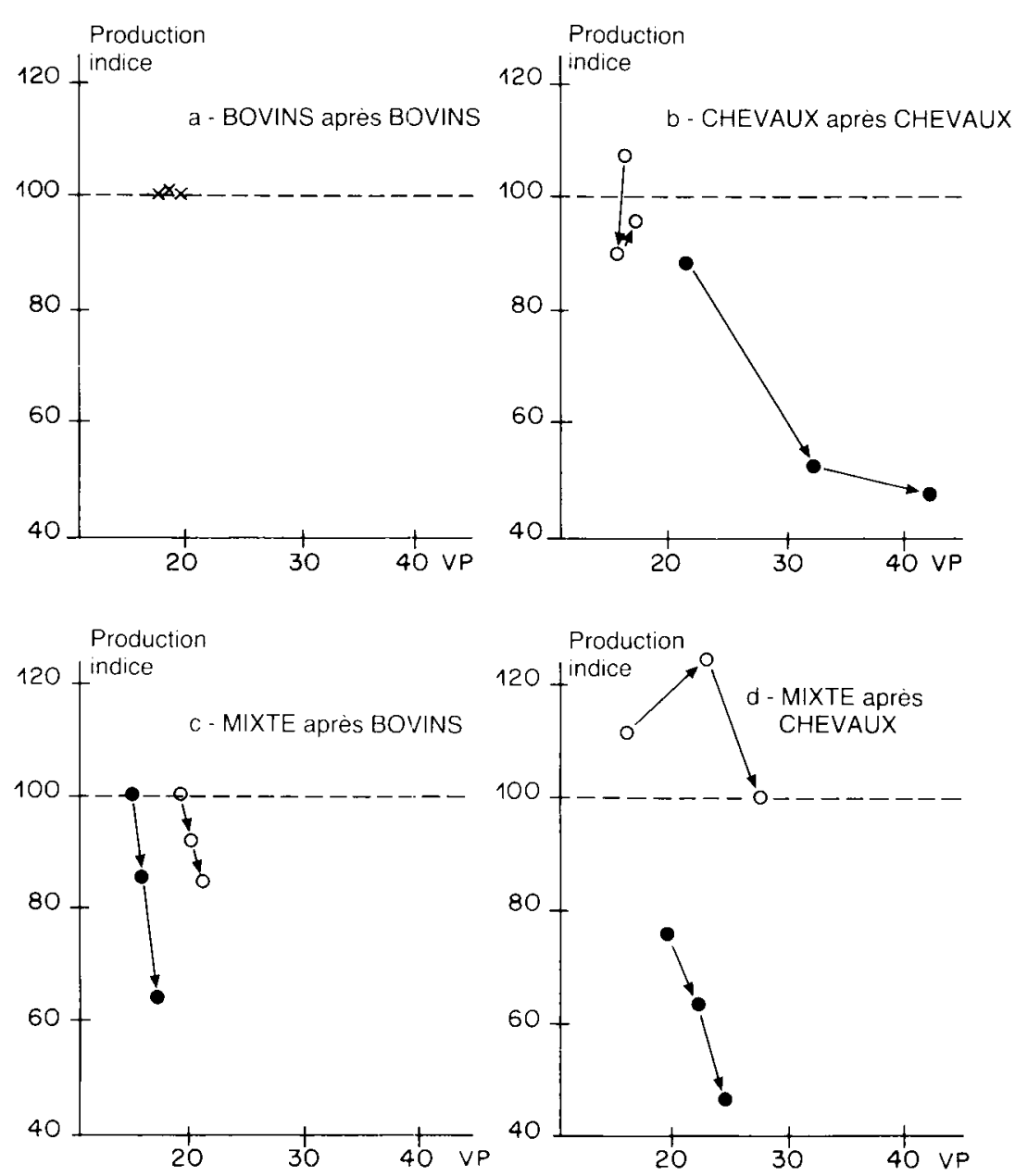

Fig. 6. Evolution de la production et de l'indice de valeur pastorale (VP) pendant les 6 dernières années. a. Bovins après bovins. b. Chevaux après chevaux. c. Mixte après bovins. d. Mixte après cheval. $O$ : chevaux, faciès refusé et fertilisé $(1980,1983$ et 1985); -: chevaux, faciès pâturé sans restitution (1980, 1983 et 1985).

bovins. De cette façon, on peut stopper l'évolution de la lande vers la pelouse à nard et mieux contrôler les ligneux, mais en contrepartie, le pâturage perdra en productivité.

\section{Alimentation des troupeaux}

La mixité doit se raisonner aussi en termes d'alimentation des troupeaux. Les résultats obtenus en pâturage monospécifique amènent la discussion sur le poids relatif de la qualité et de la quantité de fourrages offerts : quantité forte de qualité faible chez les bovins, et l'inverse chez les chevaux. Dans la mesure où les chevaux sont considérés comme des animaux plus rustiques, soit par nature, soit par leur système d'élevage, il est légitime de rechercher parmi ces deux états fourragers celui qui serait préférable pour l'alimentation des bovins. Selon les termes de Balent (1987), l'alimentation des ruminants sur leur propre pâturage fait intervenir un "tri d'impact" rapidement défavorable aux qualités ingérées; en revanche, l'alimentation des bovins sur un pâturage structuré par des chevaux fait intervenir un "choix de zone» à fortes quantités et qualité fourragères (les latrines), nécessitant peu ou moins de tri d'impact. Ce comportement est favorable aux qualités ingérées, mais les quantités ingérées dépendront de la surface propice disponible, c'est-à-dire du chargement en chevaux. Cela permet d'envisager l'existence d'une charge totale et d'un taux optimum de chevaux capables d'optimiser l'affouragement des bovins en pâturage alterné ou mixte.

\section{Conclusion}

L'expérience confirme que la remise en pâturage par les ruminants de formations végétales montagnardes abandonnées sous des chargements modérés améliore la productivité primaire sans beaucoup modifier la valeur botanique. Le comportement du cheval est complètement original 
puisque, globalement au niveau de la parcelle, il provoque les effets inverses aussi bien sur la lande que sur la pelouse (Loiseau et Martin-Rosset, 1985).

Ces résultats soulèvent en particulier deux questions. La première concerne les effets du pâturage mixte; la réponse obtenue est l'additivité des effets botaniques et la suprématie de l'effet du cheval sur la production. La deuxième question dérive de la relation inverse observée pour chaque animal entre les deux critères de jugement de la végétation; elle concerne les rôles respectifs de la qualité botanique et de la productivité pour un diagnostic opérationnel de la valeur d'usage de la végétation. Cela nécessiterait une synthèse faisant appel à la modélisation des effets animaux à partir des données expérimentales obtenues ici.

\section{Références}

Archer M. (1973) Variations in potash levels in pastures grazed by horses: a preliminary communication. Equine Vet. J. 5, 45-46

Archer M. (1977) Grazing patterns of horses. Br. Vet. J. 133,98

Archer M. (1980) Grassland management for horses. Vet. Rec. 107, 171-174
Arnold G.W. (1980) Behavioural aspects of mixed grazing. Proc. Workshop on Mixed Grazing, Galway pp. 140-153

Balent G. (1987) Structure, fonctionnement et évolution d'un système pastoral. Le pâturage vu comme un facteur écologique piloté dans les Pyrénées Centrales. Thèse, Univ. de Rennes

Carson K. \& Wood-Gush D.G.M (1983) A review of the literature on feeding, eliminative and resting behaviour. Appl. An. Ethol. 10, 165-190

De Montard F.X. (1987) Raisonnement de la fertilisation des prairies et du plan de fumure dans les exploitations d'élevage. In: Forum Fourrages Auvergne (Micol D., ed.), pp. 85-110

Edwards P.J. \& Hollis S. (1982) The distribution of excreta on New Forest grassland used by cattle, ponies and deer. J. Appl. Ecol. 19, 953-964

INRA (1979) Aspects biologiques et techniques de la remise en exploitation des hauts pâturages dégradés des Monts dore. In: Utilisation par les ruminants des pâturages d'altitude et des parcours méditerranéens. $X^{e}$ Journées du Grenier de Theix INRA pp. 57-136

Loiseau P. \& Martin-Rosset W. (1985) Utilisation des pâturages pauvres par le cheval en moyenne montagne humide. In: Vle Réunion Féd. Eur. Zoot. Kallithea

Loiseau P. \& Martin-Rosset W. (1988) Evolution à long terme d'une lande de montagne paturée par des bovins ou des chevaux. I. Condition expérimentales et évolution botanique. Agronomie 8, 161-170

Rogalsky M. (1978) Sward and soil response to grazing. In: Proc. 7th Gen. Meet. Eur. Grassl. Fed. 4, 4144 